\title{
Measurements of differential and integrated fiducial cross sections for Higgs Boson production in the four-lepton decay channel using run 2 data with the CMS detector
}

\author{
Alessandro Tarabini on behalf of the CMS Collaboration ${ }^{a}$ \\ ${ }^{a}$ Laboratoire Leprince-Ringuet, CNRS/IN2P3, Ecole Polytechnique, Institut Polytechnique de Paris, \\ Palaiseau, France, \\ Route de Saclay, 91128 PALAISEAU CEDEX \\ E-mail: alessandro.tarabini@cern.ch
}

After the discovery of the Higgs boson and its characterisation, we are entering in the precision era of the Higgs physics where we need robust measurements to spot any sign of BSM physics. Among the many available tools, fiducial cross section measurements are one of the most used in HEP due to their model independence, longevity, and simple comparison with theoretical predictions. The production cross section is measured by removing detector effects and backgrounds using an unfolding procedure.

Integrated and differential fiducial cross section measurements for the production of the Higgs boson in pp collision at the $\mathrm{LHC}$ at $\sqrt{s}=13 \mathrm{TeV}$ via the $\mathrm{H} \rightarrow \mathrm{ZZ} \rightarrow 4 \ell(\ell=\mathrm{e}, \mu)$ channel are presented. The dataset was collected by the CMS experiment in 2016, 2017, and 2018, equivalent to a validated integrated luminosity of $137 \mathrm{fb}^{-1}$.

The Ninth Annual Conference on Large Hadron Collider Physics - LHCP2021

7-12 June 2021

Online 


\section{Introduction}

The Compact Muon Solenoid (CMS) experiment [1] is one of the two multi-purpose experiments at the CERN LHC. The discovery of the Higgs boson [2-4] ended a decades-long search, but also, and perhaps more sharply, completed the set of predictions of the SM for elementary particles. However, many phenomena are still inexplicable within the SM framework, such as the inclusion of the gravitational interaction, the existence of dark matter, and the prevalence of matter over antimatter. Even some features of the SM seem strange, like its incapacity to predict particle's masses that can be determined only experimentally. Even though our knowledge about the Higgs boson properties has increased since its discovery thanks to the large amount of data collected by the ATLAS and CMS experiments, the exploration of the scalar sector is just at the beginning and remains one of the best portals to reveal uncharted territories. The LHC physics programme for the scalar sector is extremely rich and includes precision measurements of couplings, where any departure from the SM expectations would be a clear sign of new physics, measurement of the Higgs boson potential via the double Higgs boson production, searches for additional Higgs boson, rare or forbidden decays, and Dark Matter via the invisible decay. Differential cross section measurements are among the many available tools to probe the scalar sector and are one of the most used due to their potential model independence.

The decay channel of the Higgs boson in four leptons is usually referred to as golden channel. Despite suffering from limited number of events due to its small branching ratio, it features many virtues like a completely resolved final state, an excellent momentum resolution leading to a precise measurement of the Higgs boson mass, and a high signal-to-noise ratio ( 2). Three different final states are considered $(2 \mathrm{e} 2 \mu, 4 \mathrm{e}$, and $4 \mu)$ and they are regarded as three independent categories.

\section{Unfolding}

The unfolding is the core of this analysis since cross sections are measured by unfolding experimental data to the fiducial phase space at generator level (Fig. 1). The advantages of this approach are the high-longevity of the measurement, the straightforward comparison with theoretical models, and the eased combination with other fiducial results.

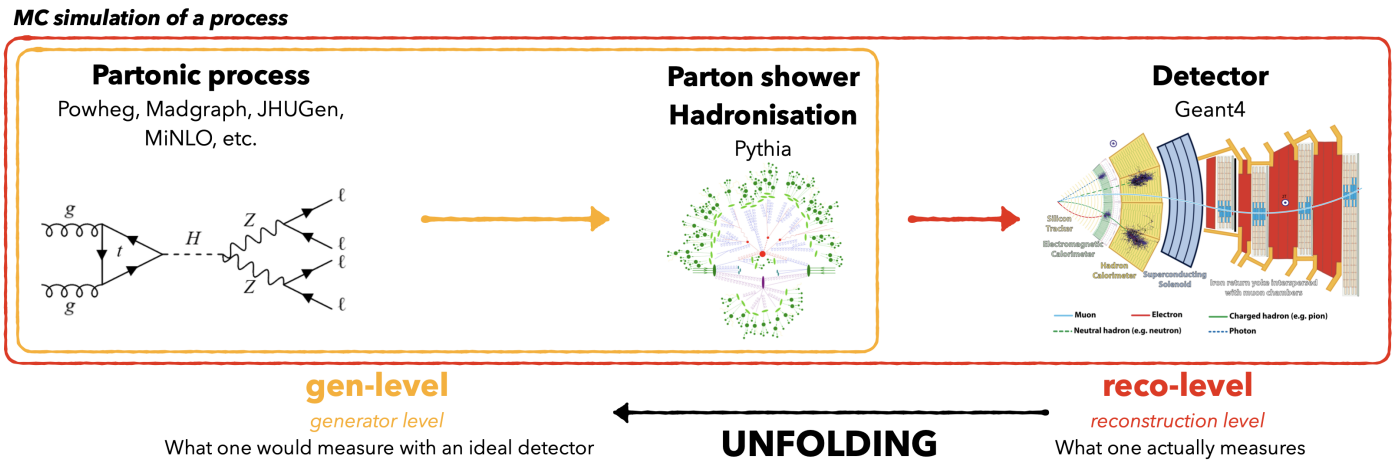

Figure 1: To produce MC predictions comparable to real data the rightward process is the one to follow. The unfolding procedure is the opposite process: starting from experimental data, which are by definition reco-level, one wants to esteem the gen-level distributions. 
The generator level is the combination of the hard partonic process and the subsequent parton shower and hadronisation, hence we aim to remove detector effects from data using an unfolding procedure. The unfolding procedure chosen in this analysis is a likelihood-based approach in which detector effects are evaluated with MC samples and embedded directly in the likelihood function used to extract the fiducial cross section.

The selection efficiencies can vary significantly between different processes of Higgs boson production and models of its properties. In order to minimise such dependence, and provide a model-independent result, the fiducial phase space is defined as a subset of the full phase space to match as closely as possible the analysis selections.

\section{Physics model}

The number of events per year $y$, final state $f$, and reco-level bin $i$ of a kinematic observable are parametrised as a function of $m_{4 \ell}$ as follows:

$$
\begin{aligned}
N_{\mathrm{obs}}^{f, i, y}\left(m_{4 \ell}\right) & =N_{\text {fid }}^{f, i, y}\left(m_{4 \ell}\right)+N_{\text {nonfid }}^{f, i, y}\left(m_{4 \ell}\right)+N_{\text {nonres }}^{f, i, y}\left(m_{4 \ell}\right)+N_{\mathrm{bkg}}^{f, i, y}\left(m_{4 \ell}\right) \\
& =\sum_{j}^{\text {genBin }} \epsilon_{i, j, y}^{f, y} \cdot\left(1+f_{\text {nonfid }}^{f, i, y}\right) \cdot \sigma_{\text {fid }}^{f, j, y} \cdot \mathcal{L} \cdot \mathcal{P}_{\text {res }}^{f, y}\left(m_{4 \ell}\right) \\
& +N_{\text {nonres }}^{f, i, y} \cdot \mathcal{P}_{\text {nonres }}^{f, y}\left(m_{4 \ell}\right)+N_{\mathrm{bkg}}^{f, i, y} \cdot \mathcal{P}_{\mathrm{bkg}}^{f, y}\left(m_{4 \ell}\right)
\end{aligned}
$$

The first two elements $N_{\text {fid }}$ and $N_{\text {nonfid }}$ are resonant contributions, namely, the selected four leptons are associated to the decay of the Higgs boson and the corresponding shape is described by a double-sided Crystal Ball function. Depending on its kinematical origin, i.e. inside or outside the fiducial phase space, an event can belong to the former or the latter term. The $\epsilon$ coefficient represents the unfolding matrix that connects the number of events at fiducial level to the number of events after the event reconstruction, encoding the information of the bin-by-bin migration brought about by detector and reconstruction effects. The non-fiducial contribution is justified by detector effects that cause differences between the quantities used for fiducial phase space definition and the analogous quantities for event selection. To target the model independece, this term is defined as a fraction of the fiducial one.

When the Higgs boson is produced through associated production, i.e. $\mathrm{VH}$ and $\mathrm{ttH}$, one or more of the four leptons might be selected among the extra leptons that are not produced via the $H \rightarrow 4 \ell$ decay process. This non-resonant contribution is empirically modelled by a Landau function.

The last term is the background contribution from irreducible ( $\mathrm{qq} \rightarrow \mathrm{ZZ}, \mathrm{gg} \rightarrow \mathrm{ZZ}$ ) processes, which is estimated from MC samples, and reducible processes $(Z+X)$, which are estimated from control regions in data.

\section{Results}

Fiducial cross sections are extracted with an unbinned maximum likelihood fit in $m_{4 \ell}$ across all years, final states, and reco-bins in the mass range $[105,140] \mathrm{GeV}$, and they are quoted both inclusively and in bins of kinematic observables. The dataset was collected by the CMS detector 

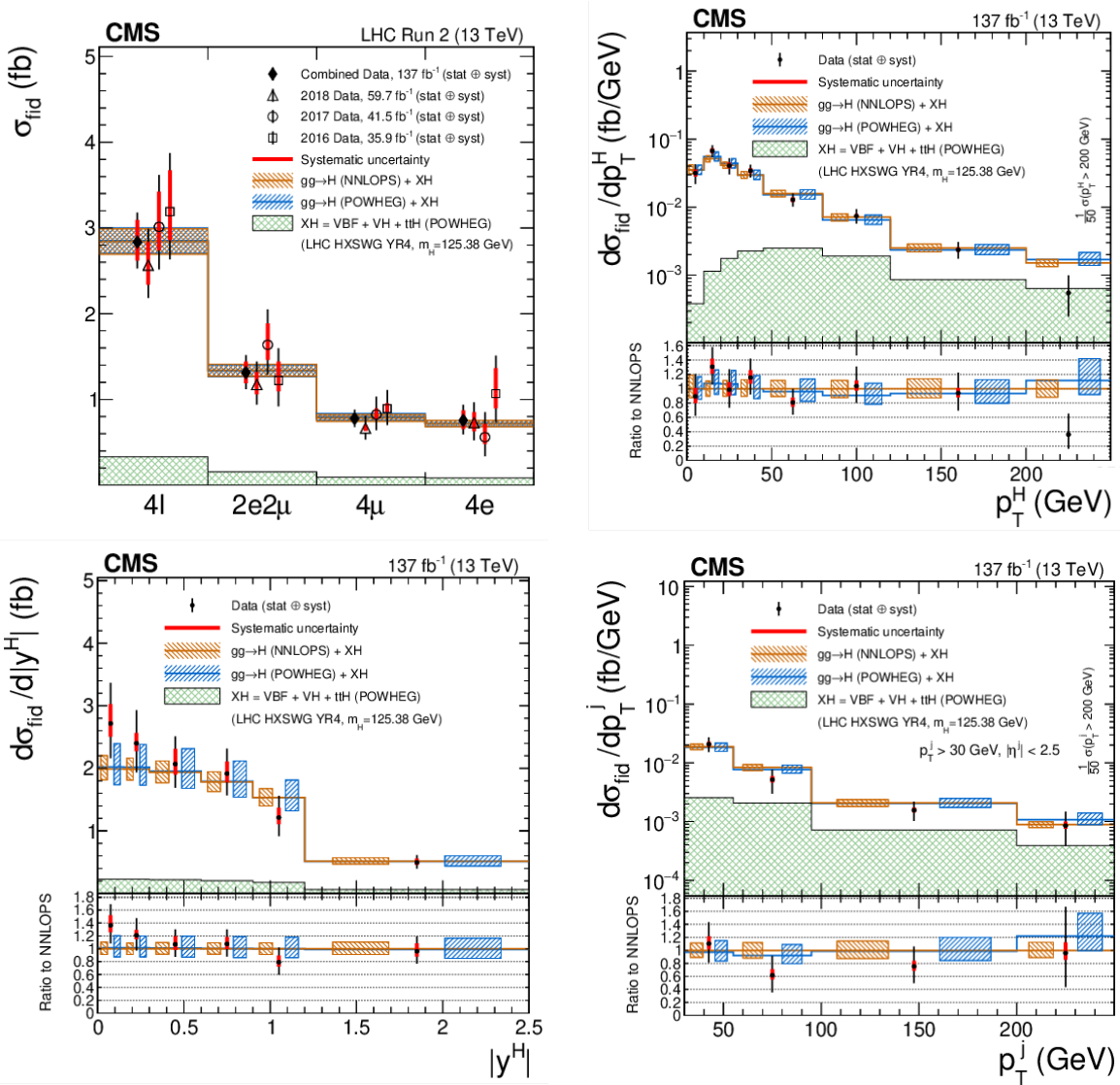

Figure 2: Inclusive and differential fiducial cross section measurements. Results are compared with SM theoretical expectations. All production modes are modelled by POWHEG, except for the leading gluon fusion process that is quoted both with POWHEG and NNLOPS. Results from [6].

during the so-called Run 2, from 2016 to 2018, equivalent to a validated integrated luminosity of $137 \mathrm{fb}^{-1}$. The inclusive result, whose $\mathrm{SM}$ expectation is $\sigma_{S M}=2.84 \pm 0.14 \mathrm{fb}$, is measured to be:

$$
\sigma_{\text {fid }}^{\text {meas }}=2.84_{-0.31}^{+0.34}=2.84_{-0.22}^{+0.23}(\text { stat })_{-0.21}^{+0.26}(\text { syst }) \mathrm{fb}
$$

The three variables reported in Fig. 2 are particularly interesting from a theoretical perspective [5]. The $p_{T}$ differential cross section is probably one of the most interesting observables since it is computed with high precision, providing an accurate comparison between data and theory, and it is sensitive to b-/c-quark Yukawa couplings [7] and Higgs boson trilinear coupling modifications [8]. The rapidity distribution is a sensitive probe to the parton distribution functions of protons and it is influenced by QCD radiative corrections. The spectrum of the $p_{T}$ of the leading jet can be used to study perturbative QCD radiation effects, Higgs boson production mechanisms, and effects of QCD resummation.

All results are in agreement with SM predictions. However, once fiducial results are provided, they can be used for further analysis by theoreticians and experimentalists to test different beyondstandard-model theories and tune SM computation, extract SM parameters (e.g. $k_{b}, k_{c}, k_{\lambda}$ ), carry out interpretations in a specific framework (e.g. EFT), and combine results with other channels and experiments [9]. 


\section{References}

[1] CMS Collaboration, "The CMS experiment at the CERN LHC", JINST 3 S08004 (2008)

[2] CMS Collaboration, "Observation of a new boson at a mass of $125 \mathrm{GeV}$ with the CMS experiment at the LHC", Phys.Lett. B716 (2012) 30-61, doi:10.1016/j.physletb.2012.02.021, arXiv:1207.7235

[3] ATLAS Collaboration, "Observation of a new particle in the search for the Standard Model Higgs boson with the ATLAS detector at the LHC", Phys.Lett. B716 (2012) 1-29, doi:10.1016/j.physletb.2012.08.020, arXiv:1207.7214

[4] CMS Collaboration, "Observation of a new boson with mass near $125 \mathrm{GeV}$ in $p p$ collisions at $\sqrt{s}=7$ and 8 TeV", JHEP 06 (2013), 081, doi:10.1007/JHEP06(2013)081, arXiv: 1303.4571 .

[5] LHC Higgs Cross Section Working Group, "Handbook of LHC Higgs Cross Sections: 4. Deciphering the Nature of the Higgs Sector", doi:10.23731/CYRM-2017-002, arXiv: 1610.07922.

[6] CMS Collaboration, "Measurements of production cross sections of the Higgs boson in the four-lepton final state in proton-proton collisions at $\sqrt{s}=13 \mathrm{TeV}$ ", Eur. Phys. J. C 81, no.6, 488 (2021), doi:10.1140/epjc/s10052-021-09200-x, arXiv:2103.04956.

[7] F. Bishara, U. Haisch, P. F. Monni and E. Re, "Constraining Light-Quark Yukawa Couplings from Higgs Distributions," Phys. Rev. Lett. 118 (2017) no.12, 121801, doi:10.1103/PhysRevLett.118.121801, arXiv:1606.09253.

[8] F. Maltoni, D. Pagani, A. Shivaji and X. Zhao, "Trilinear Higgs coupling determination via single-Higgs differential measurements at the LHC," Eur. Phys. J. C 77 (2017) no.12, 887, doi:10.1140/epjc/s10052-017-5410-8, arXiv:1709.08649.

[9] CMS Collaboration, "Measurement and interpretation of differential cross sections for Higgs boson production at $\sqrt{s}=13$ TeV", Phys. Lett. B 792 (2019), 369-396, doi:10.1016/j.physletb.2019.03.059, arXiv:1812.06504. 